\title{
Development of $\mathrm{UO}_{2}$ thermal diffusivity measurement with laser techniques
}

\author{
Thomas Doualle ${ }^{1}$, Vincent Le Guillous ${ }^{1}$,Vincent Klosek ${ }^{1}$, Claire Onofri-Marroncle ${ }^{1}$, Matthieu \\ Reymond $^{1,2}$, Laurent Gallais ${ }^{2}$, and Yves Pontillon ${ }^{1}$ \\ ${ }^{1}$ CEA, DES, IRESNE, DEC, Cadarache F-13108 Saint-Paul-Lez-Durance, France \\ ${ }^{2}$ Aix Marseille Univ, CNRS, Centrale Marseille, Institut Fresnel, Marseille, France \\ thomas.doualle@cea.fr
}

\begin{abstract}
The knowledge of the thermal conductivity of nuclear fuel and its evolution as a function of temperature and burn up is a major challenge in the context of the evaluation and understanding of irradiated fuel performances in current reactors. It is also the case for the development and qualification of fuel for future reactors. Indeed, numerical simulations of the fuel behaviour under various conditions require the accurate knowledge of thermal conductivity over a wide range of temperature (from ambient to melting point temperature) but also at the scale of few tens of micrometres to take into account the microstructural effects on the thermomechanical evolution of the fuel in normal or incidental irradiation conditions. Different methods, using laser matter interactions, can deduce the thermal conductivity from a thermal diffusivity measurement. In this paper, the potential of two techniques, which present spatial resolution from millimetre to few tens microns, are discussed in the context of the determination of the fuel thermal conductivity: laser flash method and infrared microscopy. Experiments on graphite, as material model, have been conducted and validate these two thermal diffusivity measurement techniques. We present a measurement example for both methods on graphite and then a first experiment carried out with the infrared microscopy technique on $\mathrm{UO}_{2}$.
\end{abstract}

Keywords - $\mathrm{UO}_{2}$, thermal diffusivity measurements, laser

\section{INTRODUCTION}

$I^{N}$ $\mathrm{N}$ power reactors, thermal conductivity is a key parameter for understanding nuclear fuel performance and its evolution as a function of temperature and burn up. Up to now, only few data are available in the very high temperature range (i.e. close to melting point). The laboratory reference method can deduce the thermal conductivity from a thermal diffusivity measurement. One of the most common and non-intrusive method is the laser flash method. A device employing this method has been in use at JRC Karlsruhe since 1999 [1]. This technique is based on a laser-flash excitation and infrared thermography non-contact measurement of the thermal response of a sample. The sample can be heated to different temperatures (for instance with lasers) to determine the dependence of thermal conductivity with the temperature. The thermal diffusivity measurements obtained with this technique typically have a millimeter spatial resolution. Modelling fuel behavior requires knowledge of thermal conductivity on a scale of a few tens of micrometers to take into account microstructural effects on thermomechanical behavior of the fuel under normal or off-normal conditions. To date, the flash method usually used has not provided this resolution $[1,2]$.

A laser method with few tens of microns resolution is the Infrared Microscopy technique (IRM) [3], based on the detection, by means of infrared thermography, of the sample surface temperature distribution induced by the absorption of an intensity-modulated focused laser beam.

As an example of a method which has a micrometric spatial resolution, which can be considered for our application, is the photoreflectance microscopy (PM) [4], based on the measurement and analysis of the periodic temperature increase induced by the absorption of an intensity modulated laser beam. All of these approaches, using laser-induced heat source, enable thermal diffusivity measurements for temperatures up to at least melting temperature on a scale which has never yet been reached for active materials. The potential of laser flash and the IRM methods will be discussed, in this paper. All of the presented first experiments (and associated simulations) were conducted on the ChauCoLase platform (Chauffage Controlé par Laser / laser controlled heating), available at the Institut Fresnel [5] [6]. In a first time, experiments with these two techniques were carried out on graphite, that we consider as model sample, and measurement examples will be presented. They allowed us to verify that a signal can be measured with the experimental devices envisaged, to validate the methodology and the potentiality for each techniques. The flash method was realized at different temperatures (under $1000{ }^{\circ} \mathrm{C}$ ) and we present one at $900{ }^{\circ} \mathrm{C}$ (with laser heating). For IRM, only experiments at ambient temperature have been carried out up to now. In a second time, we present in this paper a first experiment with the infrared microscopy technique on $\mathrm{UO}_{2}$. Thereafter, the idea is to extend the temperature range for both techniques and determine the dependence of thermal conductivity with the temperature. In future, the next step will be to apply the methods on non-irradiated $\mathrm{UO}_{2}$ in a glovebox in the experimental bench being developed at CEA Cadarache before its transposition in a hot cell for measurements on irradiated fuels. 


\section{THERMAL DIFFUSIVITY DETERMINATIONS: METHOD AND EXPERIMENTAL SETUPS}

This part describes the experimental benches, under development at the Institut Fresnel, implementing the two techniques previously described to obtain thermal conductivity, from thermal diffusivity measurements, with two different scales of interest: laser flash method and infrared microscopy.

The well-known technique, laser flash method, gives a global thermal diffusivity value of a sample, as a function of temperature. This method was already applied on irradiated sample in hot cell [7]. In order to go further and take into account the heterogeneity of the fuel material (like in mixed oxides fuel) and to consider the microstructural effects on thermomechanical behavior of the fuel under normal or offnormal conditions, IRM method seems suitable, and make it possible to calculate an equivalent thermal conductivity which can be used in the models.

Experiments described below have been carried out on EDM-3 graphite from POCO Graphite Inc. and $\mathrm{UO}_{2}$ samples fabricated at CEA. Graphite is a polycrystalline graphite produced by sintering and we use it as a model material to establish the methodology of the measurement techniques. The graphite and $\mathrm{UO}_{2}$ samples have a disk morphology with a diameter of $8 \mathrm{~mm}$ and thicknesses of $1.66 \mathrm{~mm}$ and $1.44 \mathrm{~mm}$ respectively. They are placed in a sample holder in a dedicated experimental chamber operated in vacuum $\left(10^{-2}\right.$ mbar $)$ which allows monitoring and optical access through multiple viewports.

\section{A. Laser Flash Technique (LF)}

During a LF experiment, the sample is subjected to a highintensity, short pulse duration. The energy is absorbed on the front surface and the thermal diffusivity value is computed from the resulting temperature response on the back surface sample, typically measured by an infrared detector. The variation in back surface temperature is referred to as a "thermogram". To determine the thermal diffusivity of graphite as a function of temperature, our approach was to set up an experimental bench to carry out experiments and measure thermograms and develop a numerical model to calculate the thermal diffusivity value, through a parameter estimation method.

The schematic diagram of the experimental setup for the LF method bench developed is given in Figure 1. A laser diode (LIMO32, Limo), operating at $880 \mathrm{~nm}$, is used to heat the sample at different temperatures. The thermal perturbation, i.e. the flash, is created by an another laser: an Ytterbium fibre laser (redPOWER Qube $2 \mathrm{~kW}$, SPI Lasers), which has a central wavelength of $1080 \mathrm{~nm}$ with a bandwidth $<4 \mathrm{~nm}$, with a maximum output power of $2000 \mathrm{~W}$. The temporal evolution of the temperature of the back face is obtained by a two-colors (channel $1: 1,65-1,8 \mu \mathrm{m}$ and channel $2: 1,45-1,65 \mu \mathrm{m}$ ) pyrometer (Metis H322, Sensortherm $\mathrm{GmbH}$ ), which has a response time of $80 \mu \mathrm{s}$.

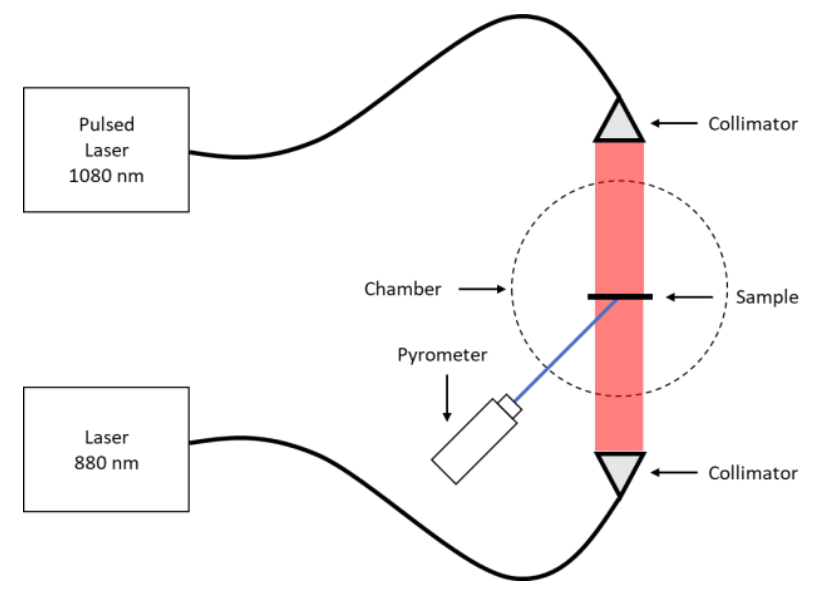

Fig. 1. Schematic of laser flash method setup

The simulations are conducted with the commercial software COMSOL which is based on the Finite Element Method in axisymmetric configuration, with a 2 dimensional meshing. The heat transfer model relies on several assumptions. First, the graphite is considered homogeneous and isotropic. Second we take into account the heat transfers by conduction combined with thermal radiation to take into account losses at the surface (convection is neglected). Third, as the energy is absorbed on the surface, there is no heat source in the material but an incoming heat flux on the boundary. The corresponding nonlinear heat transfer equation can be expressed as:

$$
\rho(T) C_{p}(T) \frac{\partial T}{\partial t}+\nabla(-k(T) \nabla T)=0,
$$

where $\rho$ is the density, $C_{p}$ is the specific heat capacity under constant pressure, $T$ is the temperature, $k$ is the thermal conductivity. The absorbed laser energy induces a heat flux $F$ expressed as:

$$
F(r, t)=A(T) . I(r, t)
$$

where $A$ is the temperature dependence absorption and $I$ the laser intensity (the measured spatial laser profile is used).

This thermal model is associated to a parameter estimation study which enables to estimate the value of one or more parameters. In our case, we calculate the value of the specific heat capacity and the thermal conductivity, so that the computational results match the experimental reference data, i.e. the thermogram. A least-squares optimization, with a Levenberg-Marquardt method, is used. It defines an objective function representing the sum of squared differences between back surface sample temperature measurements stored in an experimental data file (and acquired through the pyrometer) and corresponding expressions evaluated in the thermal model: the specific heat capacity and the thermal conductivity.

\section{B. Infrared Microscopy Method (IRM)}

Infrared Microscopy (IRM) is based on the detection of the surface sample temperature rise distribution induced by the absorption of an intensity-modulated laser beam. We assume a homogeneous, isotropic and semi-infinite material is heated at its surface by a point source with a modulated intensity at a frequency $f$. The expression of the spatial and temporal evolution of temperature $\delta T$ is [8]:

$$
\delta T(r, t)=\frac{Q}{4 \pi k t} \cdot \exp \left(-\frac{r}{\mu}\right) \cdot \cos \left(2 \pi f t-\frac{r}{\mu}\right),
$$


with $Q$ the power of the heat source, $r$ the radial distance from the heating point, $k$ the thermal conductivity of the material and $\mu$ the thermal diffusion length. The thermal diffusion length of a material is expressed as:

$$
\mu=\sqrt{\frac{a}{\pi f}}
$$

with $a$ the thermal diffusivity and $f$ the modulation frequency of the pump beam.

The equation (3) describes a wave that dampens with the increasing of the frequency $f$, as represented in Fig. 2.

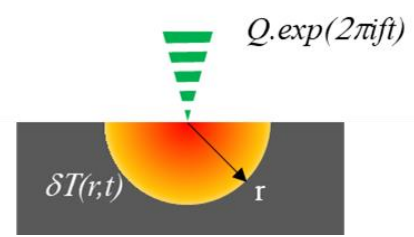

Fig. 2. Schematic representation of the spatio-temporal evolution of the temperature in a material for a source point on the surface with an intensity modulated source.

The temperature variation can be considered completely attenuated after a radial distance of approximately 3 times the thermal diffusion length. Thus, the modulated temperature of a material is confined in a volume approximately equal to 3 thermal diffusion lengths. This distance corresponds to the spatial resolution of the estimated thermal diffusivity. Therefore, by choosing an appropriate excitation frequency, it is possible to adapt the characterization scale compatible with the dimensions of the different constituents of a multi-scale (or multi-material) material. It can be seen that the phase shift of this thermal wave decreases linearly as a function of the distance $r$ from the heat source with the inverse of the diffusion length:

$$
\frac{\partial \varphi}{\partial r}=-\frac{1}{\mu}
$$

The signal phase measurement makes it possible to identify the thermal diffusivity through a linear adjustment using the Eq. (4). Thus, the slope calculation of the phase curve is a very easy way of evaluation of the thermal diffusivity by linear fit. Thus, we use an infrared camera to measure the periodic evolution of the temperature on the surface sample and deduce amplitude and phase-shift images. However, convolution effects, linked to a non-ideal point heat source, can limit the spatial resolutions of the measurement and must be taken into account to estimate accurately the thermal diffusivity.

A schematic diagram of our experimental bench is represented in Figure 3. The pump beam is a green continuous laser (Samba, Cobolt), which has a central wavelength of $532 \mathrm{~nm}$ with a bandwidth $<0.3 \mathrm{~nm}$, with a maximum output power of $1 \mathrm{~W}$. This laser wavelength was chosen to ensure efficient coupling and low penetration depth (photon energies are higher than the $\mathrm{UO}_{2}$ bandgap). The laser beam is transported through an optical fiber (PMC-400, Schäfter Kirchhoff) and focused onto the sample surface with an adjustable aspheric collimator (CFC11A-A, Thorlabs). A function generator is used to send a modulated signal to a frequency generator-driven acousto-optic modulator (MT110-A1-VIS, AA Opto-Electronic) which modulate the laser power intensity deposited on the surface of the sample. The infrared (IR) signal on the sample surface is measured to interpret the temperature distribution and is collected by a high speed IR camera (X6901SC, FLIR).

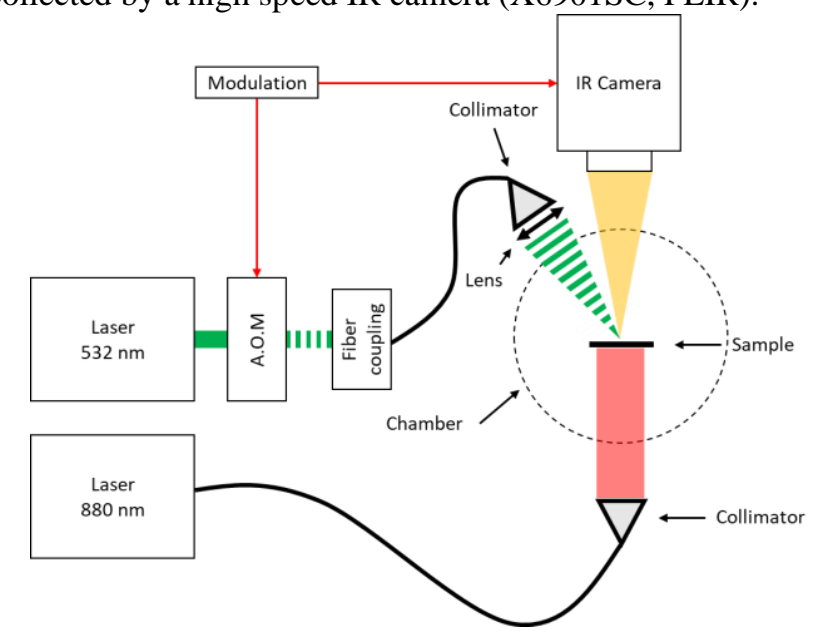

Fig. 3. Schematic of infrared microscope setup

\section{RESULTS AND DISCUSSIONS}

In this section we present the first experimental results obtained with our two thermal diffusivity measurement techniques under development. The studies were conducted on graphite sample, considered as model material. Thereafter, we show a first result with IRM technique at ambient temperature on $\mathrm{UO}_{2}$.

\section{A. Laser Flash Method}

The laser parameters are: a power of $600 \mathrm{~W}$, a pulse duration of $1 \mathrm{~ms}$ and a laser beam diameter on the sample surface of $3 \mathrm{~mm}$ (defined at $1 / \mathrm{e}^{2}$ ) with a super-Gaussian intensity profile (superGaussian function of power 4). Different initials temperatures were tested and we present below an example at around $900{ }^{\circ} \mathrm{C}$. The temperature elevation has been recorded through the pyrometer and is represented by the red dashed line in the Fig. 4. Just before the laser flash, the initial temperature on the sample was $900{ }^{\circ} \mathrm{C} \pm 10{ }^{\circ} \mathrm{C}$. We note that the temperature rise is due to the thermal diffusivity and conductivity, the amplitude is dependent on the specific heat capacity and density of the material and the temperature drop is dominated by the heat losses (convective and radiative heat transfer).

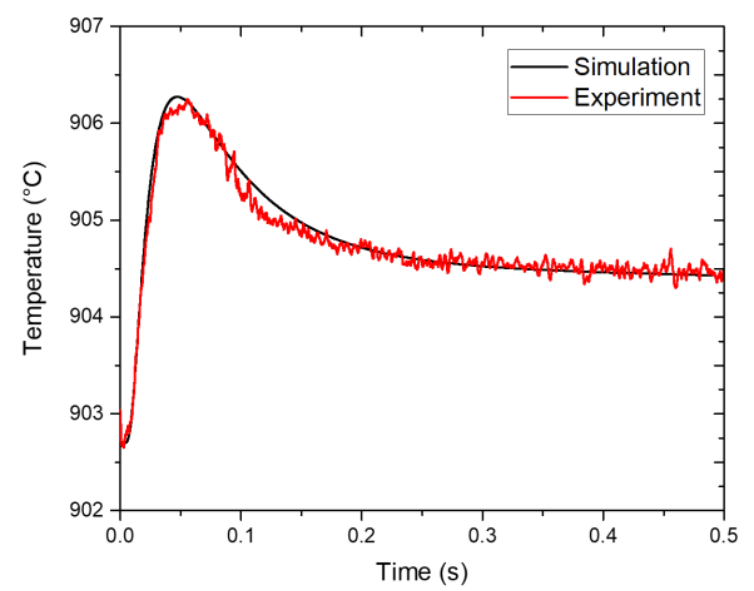

Fig. 4. On-axis surface temperature measured (red line) as a function of time and model thermogram (black line) obtained via least square optimization 
From this experimental thermogram, we have conducted the optimization algorithm to find the least squares minimum between the experiment and model thermograms. The simulated temperature elevation found is represented by the black line in Fig. 4. The maximum temperature rise of $3.5^{\circ} \mathrm{C}$, on the back surface graphite sample, is at $47 \mathrm{~ms}$ after the laser irradiation on the front surface. The Fig. 5 represents the simulated temperature distribution at this time.

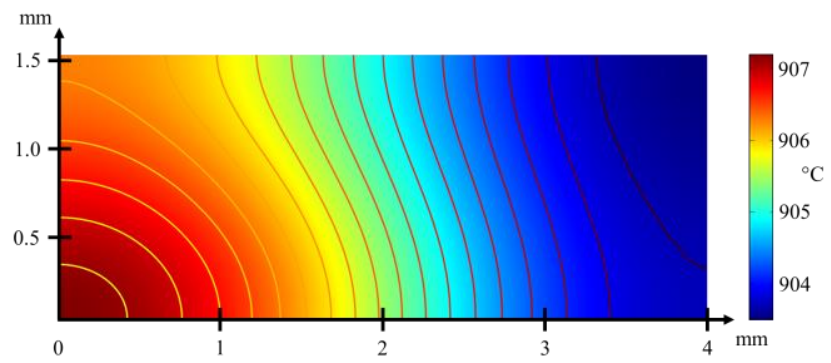

Fig. 5. Calculated temperature distribution and isotherms in graphite at $47.21 \mathrm{~ms}$

The fitted parameters thermal conductivity $k$, the specific heat capacity $C_{p}$ and the deduced thermal diffusivity, at the temperature of $900{ }^{\circ} \mathrm{C}$, are resumed and compared with the theoretical values in the Table 1.

TABLE I

COMPARISON BETWEEN THE THEORETICAL AND THE CALCULATED THERMAL PROPERTIES OF THE GRAPHITE AT $900{ }^{\circ} \mathrm{C}$

\begin{tabular}{cccc}
\multicolumn{3}{c}{ PROPERTIES OF THE GRAPHITE AT $900{ }^{\circ} \mathrm{C}$} \\
\hline \hline Property & $\begin{array}{c}\text { Theoretical } \\
{[9]}\end{array}$ & Calculated & $\begin{array}{c}\text { Relative } \\
\text { measurement } \\
\text { errors }\end{array}$ \\
\hline $\begin{array}{c}\text { Thermal } \\
\text { conductivity } \\
\left(\mathrm{W} . \mathrm{m}^{-1} \cdot \mathrm{K}^{-1}\right)\end{array}$ & 59 & 58.2 & $1.4 \%$ \\
$\begin{array}{c}\text { Specific heat } \\
\text { capacity } \\
\left(\mathrm{J}^{-1} \mathrm{~kg}^{-1} \cdot \mathrm{K}^{-1}\right) \\
\begin{array}{c}\text { Thermal } \\
\text { diffusivity } \\
\left(\mathrm{m}^{2} . \mathrm{s}^{-1}\right)\end{array}\end{array}$ & 1879 & 1955 & $4.0 \%$ \\
& $1.84 \times 10^{-5}$ & $1.75 \times 10^{-5}$ & $4.9 \%$
\end{tabular}

As we can see in the Fig. 4, the model is in very good agreement with the presented experimental thermogram example. Others initial temperatures were studied (under $1000^{\circ} \mathrm{C}$ ) and also have a concordance with theoretical values. To go further, we plan to continue experiments on graphite at higher initial temperatures to characterize the parameters as a function of the temperature in a large range and to apply the method on $\mathrm{UO}_{2}$.

Meanwhile, we will conduct a sensitivity analysis, as presented by Pavlov et al. [10], to study the relative importance of the different model parameters on the numerical parameter estimation method (sample dimensions and particularly the thickness, deposited and absorbed laser power, laser spot size, emissivity).

\section{B. Infrared Microscopy Method (IRM)}

Experiments using IRM measurement technique were conducted on the same graphite sample than in the previous section, with LF. We present below a measure that was carried out at ambient temperature. The pump laser (green laser in Fig. 3) power was $50 \mathrm{~mW}$ and the beam was focused on the surface of the sample with a beam diameter of $120 \mu \mathrm{m}$ (Gaussian distribution with diameter defined at $\left.1 / \mathrm{e}^{2}\right)$. The modulation frequency was chosen at $800 \mathrm{~Hz}$. In these conditions, the spatial resolution of the estimated thermal diffusivity is around 500 $\mu \mathrm{m}$. From two-dimensional temperature measurements obtained with the infrared camera, we deduce the phase-shift image, given in Fig. 6 (a).

The phase-shift images are used to access to the local thermal diffusivity, knowing the frequency of the modulated laser intensity. Indeed, a radial profile, as represented in Fig 6 (a) (in black line) and plotted in Fig. 6 (b), gives the phase shift of the thermal wave as a function of the radius from the center of the laser beam. The calculation of the profile slope, represented by the red dashed line in Fig. 6 (b), represents the inverse of the thermal diffusion length (Eq. 5)). To use this approach, the points to calculate the slope have to be judiciously chosen. Indeed, they have to be sufficiently far from the center of the laser beam to consider it as a point source and the number of points need to be enough to plot a representative slope and describe it accurately.

In the case presented in Fig. 6 (b), the deducted thermal diffusion length is $165 \mu \mathrm{m}$. From the Eq. (4), we are able to calculate the thermal diffusivity:

$$
a=\mu^{2} . \pi . f=6.84 \times 10^{-5} \mathrm{~m}^{2} / \mathrm{s},
$$

At ambient temperature, the theoretical value is:

$$
a(T)=\frac{k(T)}{\rho(T) \cdot C_{p}(T)}=\frac{112}{880.1737}=7.32 \times 10^{-5} \mathrm{~m}^{2} / \mathrm{s},
$$

which give a relative measurement error $E$ of $6 \%$.

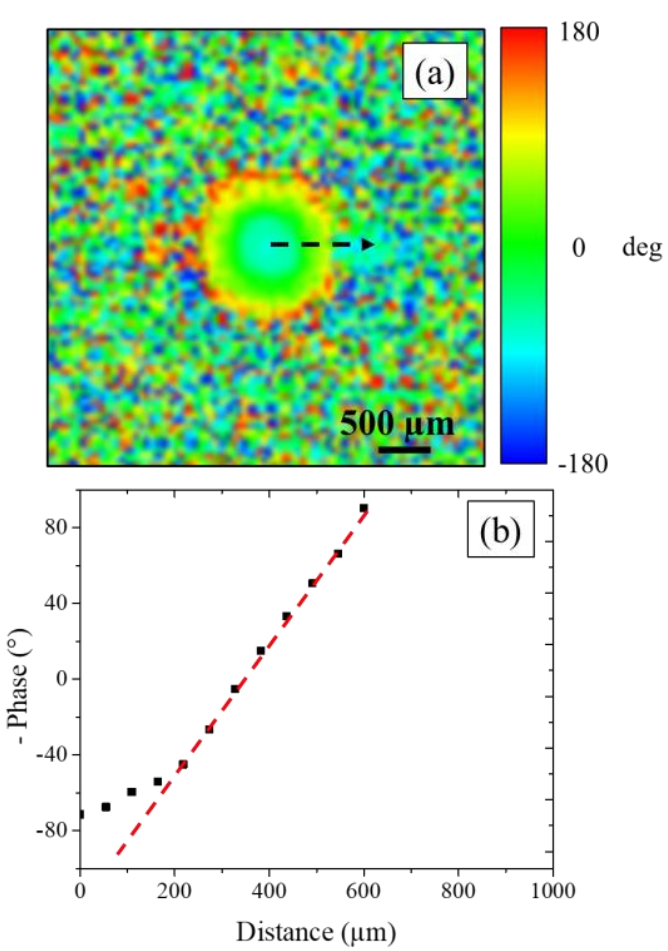

Fig. 6. (a) Phase-shift image and (b) the corresponding plot of the black dashed arrow of (a), scan at $800 \mathrm{~Hz}$. The red dashed line in (b) is the linear fit used to calculate the thermal diffusivity of the graphite.

This local thermal diffusivity measurement on graphite confirms the potential of this method. Therefore, we have carried out first IRM experiment on $\mathrm{UO}_{2}$ sample. The laser power and beam diameter are the same. The modulation frequency was chosen at $30 \mathrm{~Hz}$ which gives a spatial resolution of around $570 \mu \mathrm{m}$. The phase-shift image is calculated from the two-dimensional temperature measurement and the deduced 
thermal diffusion length is $203 \mu \mathrm{m}$, obtained from the slope. Thus, the calculated thermal diffusivity $a$ is $3.89 \times 10^{-6} \mathrm{~m}^{2} / \mathrm{s}$. At ambient temperature, the theoretical value of the $\mathrm{UO}_{2}$ thermal diffusivity is $3.42 \times 10^{-6} \mathrm{~m}^{2} / \mathrm{s}$ [11] which give an acceptable correct relative measurement error $E$ of $14 \%$.

The accuracy of our evaluation with IRM technique can be improved through different ways that will be tested in the next experiments regarding: the laser parameters (the decreasing of the laser spot size, the optimization of the laser power to enhance the signal), the IR camera performances (spatial resolution, acquisition time and frame rate) and conduct tests on other materials. Thereafter, next experiments will be carried out at different temperatures on graphite and we will continue to transfer the technique on $\mathrm{UO}_{2}$ samples. We intend to increase the spatial resolution up to few tens of microns to take into account the heterogeneity of the fuel material (like in mixed oxides fuel).

\section{CONCLUSION AND PERSPECTIVES}

This paper presents thermal diffusivity measurement techniques that we are testing in Institut Fresnel and that we consider to deploy on non-irradiated $\mathrm{UO}_{2}$ in glovebox at CEA Cadarache before its transposition in a hot cell for measurements on irradiated fuels. The idea is to reach measurements on a scale which has never yet been reached for active materials. For that, we are carrying out two different methods, using photothermal methods, enabling thermal diffusivity measurements at high temperature: the Laser Flash and the Infrared Microscopy methods for respectively a millimeter and a few tens microns resolutions. We have presented in this paper experiments on graphite samples at $900^{\circ} \mathrm{C}$ (for LF) and at ambient temperature (for IRM). A first encouraging measurement has been carried out and is presented with the infrared microscopy technique on $\mathrm{UO}_{2}$ at ambient temperature. These techniques are validated and their developments will be pursued, to have thermal properties as a function of temperature in a first time on model materials and in a second time on $\mathrm{UO}_{2}$ samples.

As a perspective of this work, we can consider an enhancement of the spatial resolution of the thermal diffusivity measurement, up to microns. A technique that would allow us to reach this resolution could be the use the Photoreflectance Microscopy (PM) [7]. The principle is similar to the IRM: a measurement and an analysis of the periodic temperature increased induced by the absorption of an intensity-modulated laser beam (pump beam). The main difference is the measurement: the local temperature increase at the sample surface is measured with the help of a secondary continuous laser beam (probe beam) through the variations of the thermally induced reflection coefficient.

\section{ACKNOWLEDGMENT}

The authors wish to thank EDF and Framatome for supporting this work in the frame of Tripartite Institute (CEA/EDF/FRA).

\section{REFERENCES}

[1] C. Ronchi, W. Heinz, M. Musella, R. Selfslag and M. Scheindlin, "A universal laser-pulse apparatus for thermophysical measurments in refractory materials at very high temperatures," International Journal of Thermophysics, vol 20, pp. 987-996, 1999, 10.1023/A:1022607924536.

[2] W. Parker, R. Jenkins, C. Butler and G. Abbot, "Flash method of determining thermal diffusivity, heat capacity and thermal conductivity," Journal of Applied Physics, vol 32, pp. 1679-1684, 1961, 10.1063/1.1728417.

[3] J. F. Bisson, "Realisation d'un diffusivimetre a haute resolution spaciale utilisant la thermographie infrarouge stimulee," M.S. thesis, Université Paris VI, 1999.

[4] J. Jumel, "Microscopie photothermique : application à la caractérisation des propriétés thermoélastiques microscopiques de composites Carbone/Carbone et des barrières thermiques," M.S. thesis, Ecole Normale Supérieure de Cachan, 2003.

[5] T. Vidal, L. Gallais, J. Faucheux, H. Capdevila, J. Sercombe and Y. Pontillon, "Simulation of Reactivity Initiated Accident thermal transients on nuclear fuels with laser remote heating, " Journal of Nuclear Materials vol 530, 151944 (2020)

[6] T. Vidal, L. Gallais, J. Faucheux, H. Capdevila and Y. Pontillon, "Using laser remote heating to simulate extreme thermal heat loads on nuclear fuels," EPJ Web of Conferences, vol 225, 08002, 2020, doi.org/10.1051/epjconf/202022508002

[7] D. Staicu, C. Cozo, G. Papaioannou, S. Bremier, V.V. Rondinella, C.T. Walker and A. Sasahara, "Thermal conductivity of homogeneous and heterogeneous MOX fuel with up to $44 \mathrm{MWd} / \mathrm{kgHM}$ burn-up", Journal of Nuclear Materials 412, pp. 129-137, 2011, doi.org/10.1016/j.jnucmat.2011.02.042

[8] D. Rochais, H. Le Houëdec, F. Enguehard, J. Jumel and F. Lepoutre, "Microscale thermal characterization at temperatures up to $1000^{\circ} \mathrm{C}$ by photoreflectance microscopy. Application to the characterization of carbon fibres," Journal of Physics D : Applied Physics 38, pp. 1498-1503, 2005, doi:10.1088/0022-3727/38/10/002

[9] Poco Graphite, Inc. "Properties and Characteristics of Graphite for the EDM Industry", Edited by R. G. Sheppard, Dwayne Morgan, D. M. Mathes, D. J. Bray, 5th Printing (2002).

[10] T. Pavlov, L. Vlahovic, D. Staicu, R.J.M. Konings, M.R. Wenman, P. Van Uffelen and R.W. Grimes, "A new C. Ronchi, W. Heinz, M. Musella, R. Selfslag and M. Scheindlin, "A universal laser-pulse apparatus for thermophysical measurments in refractory materials at very high temperatures," Thermochimica Acta, vol 652, pp. 39-52, 2017, 10.1016/j.tca.2017.03.004.

[11] J.J. Carbajo, G.L. Yoder, S.G. Popov, V.K. Ivanov, "A new C. Ronchi, W. Heinz, M. Musella, R. Selfslag and M. Scheindlin, "A review of the thermophysical properties of MOX and $\mathrm{UO}_{2}$ fuels," Journal of Nuclear Materials, vol 299, pp. 181-198, 2001, 10.1016/S0022-3115(01)00692-4 